\title{
Abstracts of the Annual Congress of the EANM, Athens, Greece, 2006
}

\author{
George P. Gerasimou • Dimitrios E. Antoniou
}

Published online: 28 March 2007

(C) Springer-Verlag 2007

\section{Eur J Nucl Med Mol Imaging (2006) 33:S65-S416}

Some of the authors' names were rendered incorrectly. The correct names are given here.

DOI 10.1007/s00259-006-0216-1, page S270

P259 In-111-DTPA-Phenyl-Pentetreotide (Octreoscan) somatostatin receptors' scintigraphy in the evaluation of patients with suspected gastro-entero-pancreatic tumors.

Gerasimou $\mathbf{G} \mathbf{P}^{\mathbf{1}}$, Aggelopoulou $\mathrm{T}^{1}$, Vasileiou $\mathrm{O}^{1}$, Liaros $\mathrm{G}^{1}$, Konidari-Dedousi $\mathrm{E}^{1}$, Basdanis $\mathrm{G}^{3}$, Prousalidis $\mathrm{I}^{3}$, Papanastasiou $\mathrm{E}^{1}$, Psarrakos $\mathrm{K}^{1}$, Dimitriadis $\mathrm{A}^{2}$, GotzamaniPsarrakou $\mathrm{A}^{1}$; 2nd Laboratory of Nuclear Medicine ${ }^{1}$, Radiological Department ${ }^{2}$, Surgical Clinic ${ }^{3}$, AHEPA University Hospital, Thessaloniki-GREECE.

Gastro-entero-pancreatic tumors (GEP) contain, in their majority, somatostatin receptors. In-111-DTPA-PhenylPentetreotide (Octreoscan) has been proved to have high affinity for somatostatin receptors subtypes 2,3 and 5 .

\footnotetext{
The online versions of the abstracts can be found at: http://dx.doi.org/10.1007/s00259-006-0213-4, http://dx.doi.org/10.1007/s00259-006-0216-1, http://dx.doi.org/10.1007/s00259-006-0217-0, http://dx.doi.org/10.1007/s00259-006-0218-z.
}

G. P. Gerasimou $(\bowtie)$

2nd Laboratory of Nuclear Medicine, AHEPA University Hospital,

1, S. Kyriakidi street, Thessaloniki 54635, Greece

e-mail: george_gerasimou@yahoo.gr

\section{E. Antoniou}

Nuclear Medicine Resident Physician,

Metaxa's Memorial Cancer Hospital,

Botassi 51, Piraeus (Athens), Greece

e-mail: dimitris.antoniou@gmail.com
The aim of the present study is to evaluate the utility of Octreoscan somatostatin receptors' scintigraphy (SRS) in the diagnosis of suspected GEP.

Thirty-three consecutive patients (15 males and 18 females-mean age 57.8 \pm 7.6) with GEP as a possible diagnosis were enrolled in the study. The primary diagnosis was diarrheic syndrome susceptive of intestinal carcinoid tumor (23 patients), carcinoid of the rectum ( 2 patients), adenocarcinoma of the pancreas ( 2 patients), insulinoma ( 2 patients), gastrinoma ( 2 patients) and hepatocellular carcinoma ( 2 patients). All patients were submitted to CT of the thorax and abdomen and Octreoascan SRS was performed 4 hours (total body and SPECT acquisition) and 24 hours (planar views), post iv injection of $185 \mathrm{MBq}$ of the radiolabeled compound.

Three of the patients with diarrheic syndrome suspect of intestinal carcinoid tumor with a positive SRS, plus one patient with adenocarcinoma of the pancreas, with a positive SRS (accumulation of the radiopharmaceutical at the place of the gallbladder), were finally diagnosed as inflammatory intestinal disease and gallbladder dilatation, respectively. At the time of the evaluation 14 of the other patients were free of disease, concerning secondary involvement. In these cases, CT and SRS studies were matched each other, with no pathological lesions and no abnormal accumulation of the radiopharmaceutical respectively. Concerning pathological cases, only one SRS study in a patient with rectum carcinoid was normal, with liver lesions in the CT study. These lesions were considered as subtypes 2,3 and 5 somatostatin receptors negative. According to these results, sensitivity of SRS study was $97 \%$ and specificity $77.8 \%$.

It is the authors' belief, that molecular imaging of somatostatin receptors with Octreoscan, is a sensitive method for the evaluation of patients with GEP tumors. However, in 
cases of intestinal disease, we should be aware of false positive results due to inflammatory processes and the presence of lymphocyte infiltration.

\section{DOI 10.1007/s00259-006-0213-4, page S145 286}

Technetium-99m human polyclonal immunoglobulin G (HIG) radionuclide study and conventional bone scan in the detection of active joints disease in patients with rheumatoid arthritis.

Gerasimou $\mathbf{G} \mathbf{P}^{\mathbf{1}}$, Aggelopoulou $\mathrm{T}^{1}$, Theodoridou $\mathrm{A}^{2}$, Konidari-Dedousi $\mathrm{E}^{1}$, Liaros $\mathrm{G}^{1}$, Vasileiou $\mathrm{O}^{1}$, Sedaghat $\mathrm{F}^{1}$, Settas $\mathrm{L}^{2}$, Gotzamani-Psarrakou $\mathrm{A}^{1}$; 2nd Laboratory of Nuclear Medicine ${ }^{1}$-Rheumatologic Department ${ }^{2}$-AHEPA University Hospital, Thessaloniki-GREECE

Radiolabelled non-specific human polyclonal immunoglobulin $(\mathrm{IgG})$ is capable of detecting pyrogen infections in patients. Tc99m-labelled polyclonal human immunoglobulin (TechneScan-HIG) can localize sterile inflammation of different joints. Rheumatoid arthritis (RA) is a chronic polyarthritis in which active inflammed joints coexist with joints in remission. The aim of this study was to evaluate the usefulness of radioscintigraphy with Tc99m-HIG in detecting active joint inflammation in patients with RA.

Twenty-four consecutive patients (4 males plus 20 females), with a mean age of $56.8 \pm 7.1$ years and duration of their disease of $24.5 \pm 4.3$ months are enrolled in the study. All patients underwent $\mathrm{x}$-ray radiological study of the affected joints, conventional bone scan with Tc-99m-HDP, 2 hours post injection of the radiopharmaceutical and 5 days later, scan with Tc-99m-HIG 4 hours post injection of the radiolabelled compound. The comparison was including uptake of both radiopharmaceuticals in different joints of the body, plus inspection of the x-rays data.

A total of 960 joints have been evaluated in the 24 patients. In 6 of the patients Tc99mHIG scan was within normal limits, matching with 2 patients with normal bone scan. All 6 patients were with inactive disease. In the 4 patients with abnormal bone scan, increased accumulation of the radiopharmaceutical has been detected in 28 joints, considered as "arthrotic" change. From the remaining 18 patients (720 joints) with an abnormal Tc99mHIG scan, increased accumulation of Tc99mHIG has been detected in 96 joints, whilst abnormal findings in bone scan have been mentioned in 118 joints, matching with 80/96 HIG abnormal joints. None of the joints with absent pain and swelling was presenting increased HIG uptake. Abnormal $\mathrm{x}$-ray findings were present only in 10 patients with disease duration more than 24 months.

In conclusion, in patients with RA, Tc99mHIG scan distinguishes joints with active disease prior to radiological findings. Active and inactive joints in RA present with increased accumulation of Tc99mHPD, in bone scanning, which cannot differentiate between joints with "arthritic" and "arthrotic" changes. Radionuclide scanning with Tc-99m-HIG is capable in identifying joints with current active inflammation.

Keywords: Technetium-99m human immunoglobulin G, rheumatoid arthritis.

Correspondence: george_gerasimou@yahoo.gr

DOI 10.1007/s00259-006-0217-0, page S296

P350 Sensitivity in the diagnosis of Alzheimer's disease using as criteria the insult of a) one and b) three of the cerebral lobes: a Tc-99m-HMPAO SPET study.

Gerasimou $\mathbf{G} \mathbf{P}^{\mathbf{1}}$, Konidari-Dedousi $\mathrm{E}^{1}$, Liaros $\mathrm{G}^{1}$, Papanastasiou $E^{1}$, Psarrakos $K^{1}$, Kosta $V^{2}$, Aggelopoulou $T^{1}$, Vasileiou $\mathrm{O}^{1}$, Sedaghat $\mathrm{F}^{1}$, Gotzamani-Psarrakou $\mathrm{A}^{1}$, Baloyannis $S^{2}$; 2nd Laboratory Nuclear Medicine ${ }^{1}-1$ st Neurologic $\mathrm{Clinic}^{2}$, AHEPA University Hospital, ThessalonikiGREECE.

Alzheimer's disease (AD) is a neurodegenerative disorder affecting people aged mainly more than 65 years. Its early diagnosis can help in a better prognosis in terms of neuroprotective treatment application. The aim of this study was to evaluate the utility of Tc-99m-HMPAO SPET study in the diagnosis of $\mathrm{AD}$.

One hundred and eight patients with a mean age of $68.9 \pm$ 7.0 years were studied fulfilling the NINDS-ADRDA criteria for the diagnosis of AD. MMSE test has been performed to all patients and 4 groups were formed: Group A, consisting of 17 patients with a very mild disturbance of the intellectual performance, who had finally developed AD (MMSE 25-28), Group B, 37 patients with mild AD (MMSE 20-25), Group C, 34 patients with moderate AD (MMSE 11-19) and group D, 20 patients with severe AD (MMSE 0-10). Comparatively, 27 normal subjects, aged $68 \pm 3.2$ years were used as controls. All subjects were submitted to cerebral blood flow (CBF) SPET study after the iv injection of $740 \mathrm{MBq}$ of Tc-99m-HMPAO. Using as criterion the onset of a) only one ("loose" criterion) and b) three of the lobes ("strict" criterion) in the temporoparietal regions in all groups plus normal subjects, sensitivity (S), positive and negative predictive value (PPV and NPV) were estimated.

Concerning SPET data from the normal subjects, only one has presented reduced $\mathrm{CBF}$ in the medial left temporal cortex, considered as false positive. Concerning patients, using as criterion, the insult of only one lobe, $\mathrm{S}$ in the diagnosis of $\mathrm{AD}$ for groups $\mathrm{A}, \mathrm{B}, \mathrm{C}$ and $\mathrm{D}$ was $53 \%$, $75.7 \%, 88.2 \%$ and $95 \%$ respectively, PPV 90\%, 96.5\%, $96.8 \%$ and $95 \%$ and the respective results for NPV were $76.5 \%, 74.3 \%, 86.7 \%$ and $96.3 \%$. Using as criterion the insult of three lobes the respective results for groups A, B, 
C, and D were $47.1 \%, 73 \%, 85.3 \%$ and $95 \%$ for $\mathrm{S}, 88.9 \%$, $96.4 \%, 96.7 \%$ and $95 \%$ for PPV and finally $74.4 \%, 72.2 \%$, $83.8 \%$ and $96.3 \%$ for NPV.

Tc-99m-HMPAO CBF study has its contribution to the diagnosis of $\mathrm{AD}$, even in doubtful cases, on respect to the clinical evaluation of the patients.

\section{DOI 10.1007/s00259-006-0217-0, page S296}

P351 Correlation of Cerebral Blood Flow SPET findings with clinical performance in patients with Alzheimer's disease.

Gerasimou $\mathbf{G} \mathbf{P}^{\mathbf{1}}$, Konidari-Dedousi $\mathrm{E}^{1}$, Liaros $\mathrm{G}^{1}$, Papanastasiou $\mathrm{E}^{1}$, Kosta $\mathrm{V}^{2}$, Vasileiou $\mathrm{O}^{1}$, Aggelopoulou $\mathrm{T}^{1}$, Sedaghat $F^{1}$, Gotzamani-Psarrakou $A^{1}$, Baloyannis $S^{2}$; 2nd Laboratory Nuclear Medicine ${ }^{1}-1$ st Neurologic Clinic $^{2}$, AHEPA University Hospital, Thessaloniki-GREECE.

Alzheimer's disease (AD) is a syndrome characterized by memory decline plus disturbances of other intellectual performances, like orientation, perception, calculation and praxis and finally speech. The aim of the present study is to compare cerebral blood flow (CBF) SPET findings in patients with $\mathrm{AD}$, with the neuropsychological tests and clinical manifestations and evaluate the clinical impact of this procedure.

Materials and Methods: One hundred and sixteen

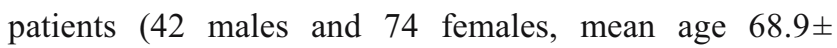
7.0 years), with $\mathrm{AD}$ were enrolled in the study. MMSE and CAMCOG neuropsychological tests have been performed to all patients. All subjects were injected with $740 \mathrm{MBq}$ of Tc99mHMPAO (Ceretec) and SPET study was performed 30 min post injection in a single-headed ADAC $\gamma$-camera equipment.

Results: Patients with mild and moderate dementia (MMSE $>20$ and between 10-20 respectively) were preceding reduced $\mathrm{CBF}$ to the temporal and parietal and/or posterior parietal cortex-symmetrical or asymmetrical-bilaterally, whilst subjects with severe dementia (MMSE $<10)$, all the above, plus reduced activity in the frontal cortex. Patients with pre-senile onset of the disease (age $<65)$ were most performing asymmetrical findings. A very significant correlation of the MMSE and CAMCOG scores with reduction of the CBF in the parietal, posterior parietal and frontal cortices bilaterally, plus in the medial and lateral aspects of the temporal cortex on the left hemisphere, was found. Memory recall has been correlated with reduced $\mathrm{CBF}$ in the medial aspect of the temporal cortices, as well as in the posterior parietal cortex bilaterally. Disturbances of perception and orientation have been correlated with reduced $\mathrm{CBF}$ in the temporal and parietal cortices, of attenuation and calculation with parietal and frontal cortices, of praxis with posterior parietal and lateral aspect of the temporal cortices and aphasia with reduced activity in the lateral temporal, frontal and parietal cortex on the left hemisphere. Treatment of the patients was re-evaluated according to the clinical plus CBF-SPET data. An heterogeneity of the findings, when correlated with the intellectual performance of the patients, can be attributed to multiple correlations and different stages of the disease.

Conclusion: CBF-SPET study correlates well with the clinical manifestations of patients with $\mathrm{AD}$ and has clinical impact, contributing to the follow-up and management.

DOI $10.1007 / \mathrm{s} 00259-006-0218-\mathrm{z}$, page S332

P528 The stunning effect is not always avoided, even if the ablation therapy is performed immediately after the $185 \mathrm{MBq}$ I-131 diagnostic whole body scan.

A. T. Georgakopoulos, S. P. Saranti, E. M. Trivizaki, D. E. Antoniou, L. A. Iordanidou, P. I. Natsis, K. M. Pateniotis, P. T. Koutsiouba; METAXA'S Memorial Cancer Hospital, Piraeus, GREECE.

Recent debates have been published about the evidence of stunning effect on the substantial I-131 ablative therapy after a diagnostic I-131 dose for DTC patients. Questions such as the existence of the stunning effect, the necessity and the recommended dose of a prior I-131 diagnostic scanning, the time interval between the diagnostic and the therapeutic dose have not been cleared yet. AIM: to determine if there is any impact of the $185 \mathrm{MBq}$ diagnostic I-131 dose on the early following ( $<45$ days) therapeutic I-131 dose and if the time interval between them affect the presence of stunning. Materials and Methods: The study included 24 patients (2 men, 22 women, mean age 46.6 years) with total/near total thyroidectomy for differentiated thyroid carcinoma. The patients were given a $185 \mathrm{MBq}$ diagnostic and a $3700 \mathrm{MBq}$ ablative I-131 dose with a time interval of 345 days between them. The patients were classified into 3 groups according to the time interval between the diagnostic and the therapeutic administration (Group A of 14 pts with a 3 days time interval, Group B of 7 pts with a 7-17 days and Group 3 of 3 patients with 30-45 days). A qualitative and a quantitative estimation were performed for the diagnostic and post therapy scanning on the 3rd and 7th day respectively.Results: Stunning phenomenon was produced totally for 14 of the $24(58.3 \%)$ patients $(9 / 14$ patients of Group A, 3/7 patients of Group B, 2/3 patients of Group C). There was not found any difference between the qualitative and the quantitative estimation of stunning effect. Statistical analysis of stunning density between the 3 groups was not possible to be done because the number of stunned patients for groups $\mathrm{B}$ and $\mathrm{C}$ was too small and this parameter remains under evaluation. Conclusions: According to our results stunning phenomenon may be produced for patients who were given a therapeutic I-131 dose in a 45 days interval from the $185 \mathrm{MBq}$ diagnostic I-131 dose. Stunning phenomenon 
could not be prevented by preserving a 72 hours time interval between the diagnostic and the ablative iodine-131 dose. Differentiation of stunning density between stunned patients with different time interval is a parameter that has to be evaluated. The possible impact of the different time interval to the therapeutic efficacy of iodine therapy for the stunned patients is one critical question that must also be evaluated.

P531 The efficacy of I-131 ablation therapy for stunned and nonstunned patients

S. P. Saranti, E. M. Trivizaki, L. Iordanidou, A. T. Georgakopoulos, D. E. Antoniou, P. Natsis, E. S. Matselas, P. T. Koutsiouba; METAXA'S Memorial Cancer Hospital, Piraeus, GREECE.

If we accept the existence of stunning, a major question that can arise concerns the incidence of this phenomenon to the I-131 therapeutic efficacy. AIM: to assess if stunning phenomenon caused by a $185 \mathrm{MBq}$ diagnostic I-131 dose has any impact upon initial I-131 ablation therapy to patients with well differentiated thyroid carcinoma (DTC). Materials and Methods: Among the patients treated with iodine- 131 the last 2.5 years, 73 DTC patients $(15$ men, 58 women, mean age 49.5 years) with total or neartotal thyroidectomy received a first ablation therapy. Between these 73 patients, the 24 -who received the ablative therapy within two months from the first diagnostic I-131 WBSwere further evaluated with post ablation scan and among them 13 produced stunning. So, based on the experienced stunning effect, we divided our patients in two groups: Group I with 60/73 nonstunned patients and group II with $13 / 73$ stunned patients. Furthermore by histology group I included 45 papillary and 15 papillary/follicular and group II included 11 papillary and 2 papillary/follicular cancers. All patients had a first $185 \mathrm{MBq}$ diagnostic I-131 WBS (DxWBS) several days to months prior to first ablation therapeutic dose and a second I-131 DxWBS was performed six months post therapy. The mean follow-up period of time was 14 months (range 7 to 30 months). By definition the patients were considered successfully treated if no functioning thyroid tissue was observed in the 6 months postablation DxWBS.Results: Ablation with the initial I-131 therapy dose was achieved in 38/60 (63.3\%) of nonstunned patients (group I) and 7/13 (53.8\%) of stunned patients (group II), an almost significant difference. The 38 single ablation dose responders of group I were 30 papillary and 8 papillary/follicular while the 7 single ablation dose responders of group II were only papillary cancers. Conclusions: According to our results there is a slight superiority of the I-131 single ablation therapy for the nonstunned patients and there is a better response of papillary cancer to the initial ablation dose. It remains to be further studied in a larger number of patients. 